\title{
Luminescence studies of HgCdTe alloys ${ }^{\text {a) }}$
}

\author{
A. T. Hunter ${ }^{\text {b) }}$ and T. C. McGill \\ California Institute of Technology, Pasadena, California 91125
}

(Received 13 November 1981; accepted 10 February 1982)

\begin{abstract}
We report the observation in $\mathrm{Hg}_{1-x} \mathrm{Cd}_{x} \mathrm{Te}$ of band-to-band, band-to-acceptor, and donoracceptor luminescence for material of $x=0.32$ and 0.5 , and bound exciton recombination luminescence, for material of $x=0.5$. The band-to-band lineshape and variation in intensity with pump power are appropriate to an electron-hole plasma with recombination proceeding without wave vector conservation. Differences between the spectra among the four 0.5 samples studied are attributed to variations in the $\mathrm{Hg}$ vacancy concentration. Shifts in luminescence energy across one of the $x=0.32$ samples imply a change in composition across the surface of the sample of 0.03 $\mathrm{cm}^{-1}$. The absence of bound exciton luminescence in the $x=0.32$ samples is consistent with theoretical calculations by Osbourn and Smith showing a radiative efficiency of $20 \%$ for bound excitons in material of this composition and $90 \%$ for $x=0.48$ material. From line shape separations, we estimate acceptor binding energies of $14.0 \pm 1.0$ and $15.5 \pm 2.0 \mathrm{meV}$ in $x=0.32$ and 0.48 materials, respectively, and donor binding energies of $1.0 \pm 1.0$ and $4.5 \pm 2.0 \mathrm{meV}$, respectively.
\end{abstract}

PACS numbers: 78.55.Hx, 71.35. + z, 71.55.Dp

\section{INTRODUCTION}

Photoluminescence spectra have been widely used as a method for studying the properties of II-VI semiconductors. ${ }^{1}$ However, the photoluminescence spectra for $\mathrm{Hg}_{1-x} \mathrm{Cd}_{x} \mathrm{Te}$ has not been explored extensively. ${ }^{2,3}$ Recently we have initiated a study of the photoluminescence spectra of $\mathrm{HgCdTe}$ with $x$ values less than $0.5 .^{4}$

In this manuscript we summarize a series of luminescence experiments that we have carried out on $\mathrm{HgCdTe}$. Spectra were taken on bulk-grown samples of $x=0.32$ and $x=0.48$, and on an epitaxial sample with $x=0.51$.

\section{EXPERIMENTAL TECHNIQUE}

Spectra were taken over the temperature range of 5 to 30 $\mathrm{K}$ for a wide range of pump-laser power densities. A mechanically chopped $\mathrm{Ar}^{+}$ion laser was used to create excited electrons and holes in the samples. The samples were mounted in a cryogenic dewar. The light emitted by the sample was focused onto the slits of a grating spectrometer and was detected with a solid-state detector. The slit width used for most of the spectra gave an energy resolution of $\sim 1 \mathrm{meV}$. An InAs detector was used for the $x=0.48$ and 0.51 material, and an InSb detector was used for the $x=0.32$ material. The detector output was fed into a lock-in amplifier, with a signal from the mechanical chopper used as a reference signal.

\section{RESULTS}

Figure 1 is a spectrum showing luminescence intensity as a function of emitted photon energy for Sample 1 which has a composition of $x=0.48$. This sample is $n$ type, with $N_{D}-N_{A}=10^{15} \mathrm{~cm}^{-3} .^{5}$ This spectrum shows all of the different luminescence lines we have observed in the material. The line at the highest energy in the figure has the characteristics of band-to-band recombination luminescence. We at- tribute the line at the lowest energy to donor-acceptor recombination below $\sim 10 \mathrm{~K}$, and to free electron-to-bound hole recombination at higher temperatures where a substantial fraction of the donors are ionized. The line at the intermediate energy has the characteristics of bound exciton recombination luminescence.

The shift of the line peaks with temperature provides much of the information necessary to identify the mechanisms responsible for the luminescence. Figure 2 summarizes the peak positions for the sample of Fig. 1 for a variety of temperatures and pump powers. The points for a given

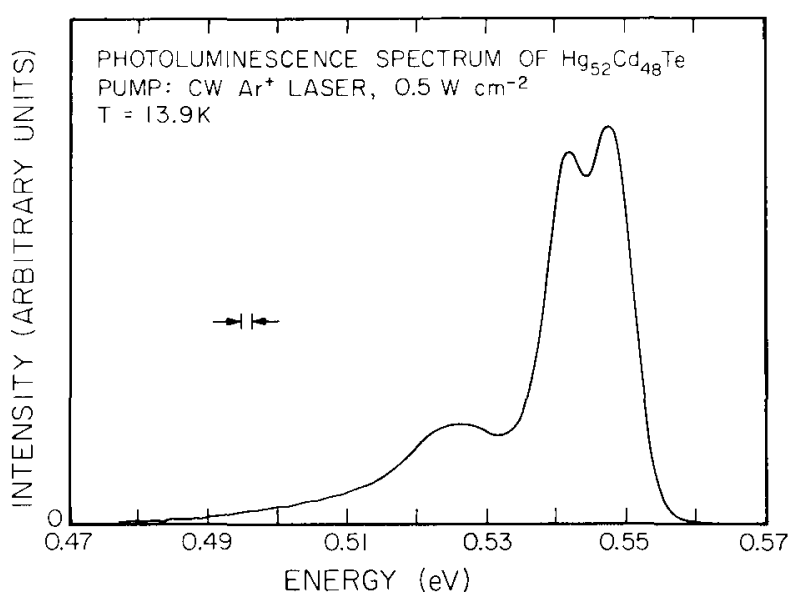

FIG. 1. Spectrum of $\mathrm{Hg}_{0.52} \mathrm{Cd}_{0.48} \mathrm{Te}$ showing photoluminescence intensity vs photon energy with the sample at $13.9 \mathrm{~K}$. The high energy peak is due to band-to-band recombination. The low energy peak is due to a combination of donor-acceptor and band-to-acceptor luminescence. The intermediate energy line is due to bound exciton luminescence. The sample was $n$-type, with $N_{D}-N_{A} \sim 10^{15} \mathrm{~cm}^{-3}$. 
band at one pump power are connected together as an aid to the eye. The band-to-band peak shifts toward higher energy at a rate equal to $3 k_{B}$, where $k_{B}$ is Boltzmann's constant. The bound exciton, on the other hand, does not shift at all within the uncertainty of the measurement. The low energy impurity related line shifts very little below $10 \mathrm{~K}$, especially at the two lowest pump powers. Then it shifts rapidly above $10 \mathrm{~K}$.

The peak shift of the band-to-band line is consistent with an electron-hole plasma recombining without wave vector conservation. This mechanism predicts a line peak which shifts, in the nondegenerate limit, at a rate equal to $2 k_{B}$. While this is not quite enough to match the shift observed, it is a better match than could be obtained with other mechanisms. For example, an electron-hole plasma in which the wave vector is conserved during recombination would shift at a rate of only $\frac{1}{2} k_{B}$. A shift of the band gap with temperature may explain some of the additional shift. Another piece of evidence for recombination without wave vector conservation is that the predicted lineshape fits the data reasonably well at $30 \mathrm{~K}$. The carrier density was the only parameter varied which affected the shape and width of the fit, and the value of the carrier density needed to fit the observed width of the line was consistent with that estimated from the pump-laser power density.

The factors that identify the intermediate energy line as bound exciton luminescence are the lack of shift as temperature is varied and a rapid decrease in intensity as temperature is raised. Since the peak does not shift with temperature, the electron and hole involved in the recombination must be bound. However, the rapid decrease in intensity with respect to the other lines indicate that the binding energy is small. Both of these properties are characteristic of bound exciton recombination luminescence.

The lack of shift of the low energy peak at low temperatures again indicates that bound particles are involved in the recombination. However, the luminescence is deeper than for the bound exciton line, and the line width is much greater, pointing to donor-acceptor pair recombination. The rapid shift in energy of the line peak above $10 \mathrm{~K}$ comes about

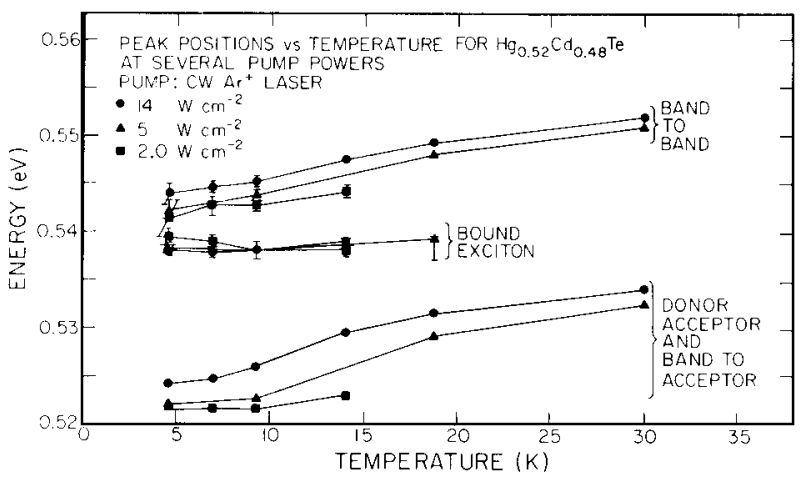

FIG. 2. Summary of luminescence band peak energies vs temperature of $\mathrm{Hg}_{0.52} \mathrm{Cd}_{0.48} \mathrm{Te}$ at three different pump powers for the sample of Fig. 1 . The line between data points are drawn as a visual aid, and connect points measured at different temperatures for one type of luminescence band at one pump power. Error bars are given where significant; the uncertainty is due to overlap between bands. because the weakly bound donors become thermally ionized. The widths of the donor-acceptor luminescence and the free-electron to bound-hole luminescence lines are too broad compared to the donor binding energy to resolve each as a separate line.

Three other samples of $x=0.5$ material were studied. The results of the luminescence experiments are similar for these samples as for the sample described above, but there are a few differences in some of the samples. Sample 2 was $p$ type with $N_{A}-N_{D} \sim 10^{15} \mathrm{~cm}^{-3}$, sample 3 was $p$ type with $N_{A}-N_{D} \sim 10^{16} \mathrm{~cm}^{-3}$, and sample 4 was an epitaxial sample grown on a CdTe substrate. ${ }^{5}$ The bound exciton was observed in samples 2 and 4 but was not observed in sample 3 . The other luminescence lines were observed in all the samples.

Sample 3, in addition to showing no bound exciton luminescence, also had a very weak band-to-band line in comparison to sample 1 , under similar pump-laser power densities and temperatures. This difference could be explained by a higher defect density in sample 3 than in sample 1. The added defects could directly cause the difference by being the acceptor responsible for the luminescence, or indirectly by reducing the free carrier lifetime by promoting nonradiative recombination. $\mathrm{Hg}$ vacancies are a likely candidate for the defect. Evidence for this explanation is provided by the evolution of the spectrum from sample 2. Originally, the sample showed a strong bound exciton line at low temperature. Prior to many of the luminescence experiments on the sample, its surface was etched briefly in a weak bromine-methanol solution, since this procedure was found to enhance the luminescence signal. After a period of several months, and several etchings, the bound exciton signal was no longer observed, and the luminescence signal resembled that observed from sample 3 . All of the bulk grown samples were annealed in a $\mathrm{Hg}$ atmosphere after growth, which reduced the carrier concentration in a thin surface layer, ${ }^{5}$ presumably by reducing the $\mathrm{Hg}$ vacancy concentration. Therefore, it is likely that

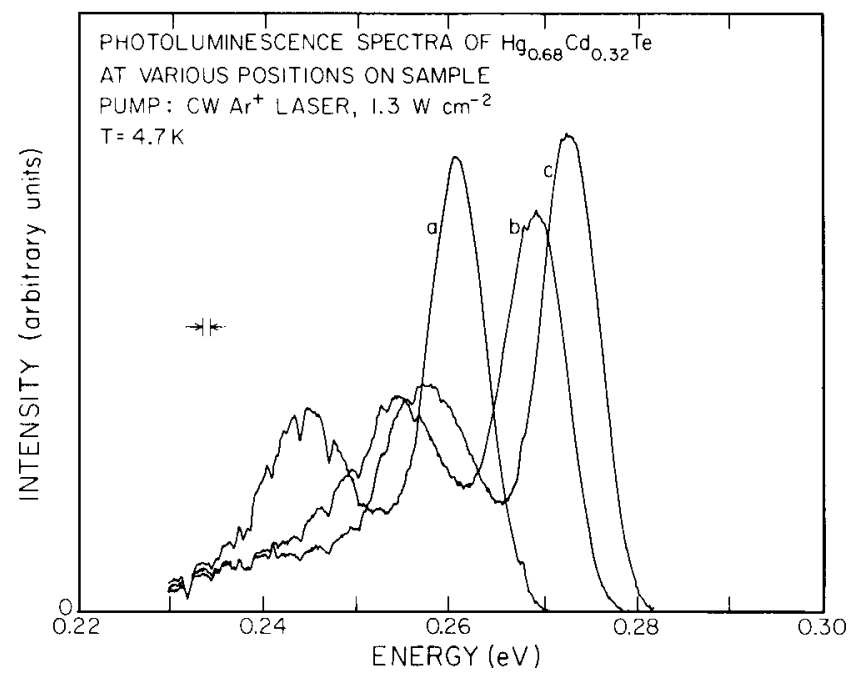

FIG. 3. Spectra of $\mathrm{Hg}_{0.68} \mathrm{Cd}_{0.32}$ Te showing photoluminescence intensity vs photon energy. For spectrum (b), the pump laser was $1.0 \mathrm{~mm}$ above its position for spectrum (a), and for (c), $2.5 \mathrm{~mm}$ above its position for spectrum (a). The shift in energy between (a) and (c) implies a shift in composition for $0.03 \mathrm{~cm}^{-1}$. 
the interior of sample 2 (and all of the other bulk-grown samples) had a much higher $\mathrm{Hg}$ vacancy concentration, than the region near the surface. Therefore, the etchings gradually exposed material with a higher vacancy concentration, which changed the luminescence signal. The similarity between the spectra in sample 3 and the spectra in sample 2 after etching indicates that sample 3 had a higher vacancy concentration to begin with, which was responsible for the dominance of the defect related band.

The spectra observed from sample 4, the epitaxial sample, were similar to those observed from sample 1 and from sample 2 before etching. However, the band-to-band line was much more intense at comparable pump power densities than in the other two bulk-grown samples. This may indicate a much lower overall defect density for the epitaxial sample.

The data for the $x=0.32$ material is shown in Fig. 3. The band-to-band and electron-to-bound hole lines are observed in this material. This figure shows luminescence spectra from a $p$-type $x=0.32$ sample, with $N_{A}-N_{D} \sim 10^{15} \mathrm{~cm}^{-3.5}$. The three spectra shown in this figure were all taken at the same pump power and temperature. The only thing varied between the spectra was the position of the pump beam on the surface of the $\mathrm{HgCdTe}$ wafer. Using measured relationship between composition and band gap, ${ }^{6}$ the composition gradient is estimated to be $0.03 \mathrm{~cm}^{-1}$ for this sample.

The major difference between the $x=0.5$ samples and the $x=0.32$ samples is that the bound exciton luminescence line was not seen in either of the two $x=0.32$ samples studied. One possible explanation for this absence is provided by a calculation of the radiative efficiency of bound exciton luminescence. ${ }^{7}$ This calculation shows that the radiative efficiency in $x=0.32$ material is $20 \%$, much smaller than the $90 \%$ radiative efficiency for bound exciton luminescence in $x=0.48$ material.

The energy differences between the lines allow estimates of the donor and acceptor binding energy. From the separation between the band-to-band line and the band-to-boundhole line, we estimate $14.0 \pm 1.0$ and $15.5 \pm 2.0 \mathrm{meV}$ in $x=0.32$ and 0.48 material, respectively, for the acceptor binding energies. Values of $1.0 \pm 1.0$ and $4.5 \pm 2.0$, respectively, were obtained for the donor binding energies from the energy difference between the donor-acceptor and band-tobound hole line positions.

\section{CONCLUSIONS}

In summary, we have observed luminescence from bandto-band transitions, donor-acceptor transitions, and free electron-to-bound-hole transitions in $x=0.32$ and $x=0.5$ $\mathrm{Hg}_{1-x} \mathrm{Cd}_{x} \mathrm{Te}$. We have observed bound exciton recombination luminescence in $x=0.5$ material. The variation in composition across the surface of a sample was measured using photoluminescence. Finally, donor and acceptor binding energies were estimated from the data. A more extensive report of this work is contained in Ref. 4.

\section{ACKNOWLEDGMENTS}

We wish to acknowledge P. Bratt and K. Riley of the Santa Barbara Research Center for providing us with HgCdTe samples, and L. DeVaux of the Hughes Research Laboratories for useful discussions on the properties of $\mathrm{HgCdTe}$.

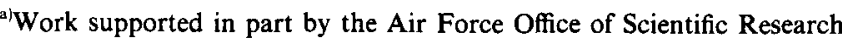
under Grant No. AFOSR-77-3216.

${ }^{b}$ Present address: Hughes Research Laboratories, 3011 Malibu Canyon Road, Malibu, CA 90265.

'See, for example, A. A. Bergh and P. J. Dean, Light-Emitting Diodes (Clarendon, Oxford, 1976), p. 245ff; and references therein.

${ }^{2}$ C. T. Elliot, I. Melngailis, T. C. Harman, and A. G. Foyt, J. Phys. Chem. Solids 33, 1527 (1972).

${ }^{3}$ V. I. Ivanov-Omskii, V. A. Mattseva, A. D. Britov, and S. D. Sivachenko, Phys. Status Solidi A: 46, 77 (1978).

${ }^{4}$ A. T. Hunter and T. C. McGill, J. Appl. Phys. 52, 5779 (1981).

${ }^{5} \mathrm{P}$. Bratt (private communication).

${ }^{6} \mathrm{R}$. Dornhaus and G. Nimtz, in Springer Tracts in Modern Physics, edited by G. Hohler (Springer, Berlin, 1976), Vol. 78, pp.1-112.

${ }^{7}$ G. C. Osbourn and D. L. Smith, Phys. Rev. B 20, 1556 (1979).
} 